International Journal of Pure and Applied Mathematics

Volume 108 No. 4 2016, 945-955

ISSN: 1311-8080 (printed version); ISSN: 1314-3395 (on-line version)

url: http://www.ijpam.eu

doi: 10.12732/ijpam.v108i4.19

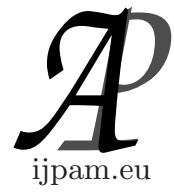

\title{
DOUBLE POWER METHOD ITERATION FOR PARALLEL EIGENVALUE PROBLEM
}

\author{
K. Rhofir ${ }^{1}$, M. Ameur ${ }^{2}$, A. Radid ${ }^{3}$ \\ ${ }^{1}$ University Hassan 1-er LISERT-ENSA Bd. \\ Beni Amir, BP. 77, Khouribga, MOROCCO \\ ${ }^{2}$ University Kaddi Ayad \\ ENSA Av Abdelkrim Khattabi, BP 575 \\ Gueliz-Marrakech, MOROCCO \\ ${ }^{3}$ University Hassan II Casablanca \\ FSAC-MACS, BP. 5366, Maarif Casablanca, MOROCCO
}

\begin{abstract}
In this paper, we introduce the double power iteration method witch can be seen as an extension of the classical power iteration in the sense that we calculate the two dominants eigenvalues at each stage. This work aims to propose a solution of slow convergence problem to the power iteration method and the calculation of the second dominant eigenvalue. We develop a parallel iterative procedure for the calculation of eigenvalues of a given matrix and we can expressed this method as a Quadrant Interlocking Factorization (QIF) which introduced by [7] and studied by [8]-[9] in other works.
\end{abstract}

AMS Subject Classification: 15A06, 15A23

Key Words: double power iteration, Q.I.F Fcatorization, RL, LU, QR Factorization, linear system and parallel process

\section{Introduction}

Although the power iteration method approximates only one eigenvalue of a matrix, it remains useful for certain computational problems. For instance, Google uses it to calculate the PageRank of documents in their search engine [1], and Twitter uses it to show users recommendations of who to follow [2].

Received: June 13, 2016

Published: August 16, 2016

${ }^{\S}$ Correspondence author
(C) 2016 Academic Publications, Ltd.

url: www.acadpubl.eu 
For matrices that are well-conditioned and as sparse as the Web matrix, the power iteration method can be more efficient than other methods of finding the dominant eigenvector. But when the two dominants eigenvalues are of approximately the same magnitude this method may converge slowly or fail.

The central to the field of matrix computations is the problem of solving a system of linear equations $A x=b$ and the numerical solutions of the eigenvalues and the corresponding eigenvectors of a large and dense matrix plays an important role in numerous scientific applications. Rutishauser [5] proposed the LR algorithm for the calculation of the eigenvalues of a $A$. In this procedure, we obtain a sequence of matrices $A^{(1)}, A^{(2)}$, . . . which in general reduces to an upper triangular matrix. The most popular methods developed to solve this problem after [5] by factorization such as the QR algorithm, the Givens method, the Housholder transformation [10] [11] and by projection techniques on appropriate subspaces such as Lanczos and Davidson methods [13] [12].

In this paper, we present a double power iteration as iterative procedure to calculate the two dominants eigenvalues at each stage. This procedure still a variants of LR algorithm and the eigenvalues are obtained from simple $2 \times 2$ matrices derived from the main and cross diagonals of the limit matrix. So we can compute the eigenvalues of this limit matrix in parallel. We can express our approach as a Quadrant Interlocking Factorization and then we can give the solution of the linear equation $A x=b$.

The paper is organized as follows. We start by giving mathematical framework for double power iteration algorithm for computing the two dominant eigenelements of a matrix $A$. Next, we prove the convergence of the algorithm. Finally, we factorize $A$ in the form like those in [7] to evaluate all eigenvalues and we give an easy way to the solution of linear equation.

\section{Double Power Iteration Method}

Let $A \in \mathcal{L}\left(\mathbb{R}^{n}\right)$ be a real matrix, $I$ the identity matrix and $\left\{e_{i}\right\}_{1 \leq i \leq n}$ the standard basis of $\mathbb{R}^{n}$. We denote by $u^{t}$ the transpose of vector $u, B^{T}$ the transpose of matrix $B$ and by $\delta_{i j}$ for $i, j \in\{1, \ldots, n\}$, the Kronecker delta

$$
\delta_{i j}=\left\{\begin{array}{ll}
1 & i=j \\
0 & i \neq j
\end{array} .\right.
$$

The proposed double power iteration method is the solution of the following problem: 
Find $x, y, z$ and $t$ the real elements such that: for $u$ and $v$ two vectors on $\mathbb{R}^{n}$, we have

$$
\left\{\begin{array}{l}
A u=x u+y v \\
A v=z u+t v
\end{array}\right.
$$

This problem is equivalent to transform

$$
A=\left(\begin{array}{ccccc}
a_{1,1} & \cdots & a_{1, i} & \cdots & a_{1, n} \\
\vdots & \ddots & & . & \vdots \\
a_{i, 1} & & a_{i, i} & & a_{i, n} \\
\vdots & . & & \ddots & \vdots \\
a_{n, 1} & \cdots & a_{n, i} & \cdots & a_{n, n}
\end{array}\right)
$$

into

$$
A^{(1)}=\left(\begin{array}{ccccc}
a_{1,1}^{(1)} & \cdots & a_{1, i}^{(1)} & \cdots & a_{1, n}^{(1)} \\
0 & \ddots & & . \cdot & 0 \\
\vdots & & a_{i, i}^{(1)} & & \vdots \\
0 & . \cdot & & \ddots & 0 \\
a_{n, 1}^{(1)} & \cdots & a_{n, i}^{(1)} & \cdots & a_{n, n}^{(1)}
\end{array}\right)
$$

Let define a matrix $Q$ applied for this transformation by $Q=I+u \cdot e_{1}^{t}+v \cdot e_{n}^{t}$ where $u_{1}=u_{n}=0$ and $v_{1}=v_{n}=0$.

Then $Q^{-1}=I-u \cdot e_{1}^{t}-v \cdot e_{n}^{t}$.

So we write $A^{(1)}$ in the form $A^{(1)}=Q^{-1} A Q$.

Then for $1 \leq i, j \leq n$, we have:

$$
\begin{gathered}
a_{i, j}^{(1)}=<A^{(1)} e_{j}, e_{i}>=<Q^{-1} A Q e_{j}, e_{i}>=<A Q e_{j},\left(Q^{-1}\right)^{T} e_{i}> \\
=<A\left(I+u \cdot e_{1}^{t}+v \cdot e_{n}^{t}\right) e_{j},\left(I-u \cdot e_{1}^{t}-v \cdot e_{n}^{t}\right) e_{i}> \\
=<A\left(e_{j}+\delta_{1 j} u+\delta_{1 n} v\right), e_{i}-u_{i} e_{1}-v_{i} e_{n}> \\
=a_{i, j}-u_{i} a_{1, j}-v_{i} a_{n, j}+\delta_{1 j}\left(<A u, e_{i}>-u_{i}<A u, e_{1}>-v_{n}<A u, e_{n}>\right) \\
+\delta_{n j}\left(<A v, e_{i}>-u_{i}<A v, e_{1}>-v_{i}<A v, e_{i}>\right)
\end{gathered}
$$

By (2.2), we have

$$
\forall i, 1<i<n a_{i, 1}^{(1)}=a_{i, n}^{(1)}=0
$$


then, for $j=1$ and $j=n$, we get the following system:

$$
\begin{gathered}
(E)\left\{\begin{array}{l}
a_{i, 1}-u_{i} a_{1,1}-v_{i} a_{n, 1}+<A u, e_{i}>-u_{i}<A u, e_{1}>-v_{i}<A u, e_{n}>=0 \\
a_{i, n}-u_{i} a_{1, n}-v_{i} a_{n, n}+<A v, e_{i}>-u_{i}<A v, e_{1}>-v_{i}<A v, e_{i}>=0
\end{array}\right. \\
(E)\left\{\begin{array}{l}
u_{i}\left(a_{1,1}+<A u, e_{1}>\right)+v_{i}\left(a_{n, 1}+<A u, e_{n}>\right)=a_{i, 1}+<A u, e_{i}> \\
u_{i}\left(a_{1, n}+<A v, e_{1}>\right)+v_{i}\left(a_{n, n}+<A v, e_{n}>\right)=a_{i, n}+<A v, e_{i}>
\end{array}\right.
\end{gathered}
$$

Let:

$$
\begin{aligned}
& \bar{u}=u+e_{1} \\
& \bar{v}=v+e_{n}
\end{aligned}
$$

Then $\forall i, 1 \leq i \leq n$, we have:

$$
(E)\left\{\begin{array}{l}
\bar{u}_{i}<A \bar{u}, e_{1}>+\bar{v}_{i}<A \bar{u}, e_{n}>=<A \bar{u}, e_{i}> \\
\left.\bar{u}_{i}<A \bar{v}, e_{1}>\right)+\bar{v}_{i}<A \bar{v}, e_{n}>=<A \bar{v}, e_{i}>
\end{array}\right.
$$

so

$$
(E)\left\{\begin{array}{l}
\bar{u}<A \bar{u}, e_{1}>+\bar{v}<A \bar{u}, e_{n}>=A \bar{u} \\
\left.\bar{u}<A \bar{v}, e_{1}>\right)+\bar{v}<A \bar{v}, e_{n}>=A \bar{v}
\end{array}\right.
$$

Assume that the matrix $A$ is diagonalizable, then there exist a basis $\left\{z_{i}\right\}_{1 \leq i \leq n}$ formed by the eigenvectors of $A$ such that:

$$
\begin{aligned}
\bar{u} & =\sum_{i=1}^{n} \alpha_{i} z_{i} \\
\bar{v} & =\sum_{i=1}^{n} \beta_{i} z_{i}
\end{aligned}
$$

We get then,

$$
\begin{gathered}
(E)\left\{\begin{array}{c}
\sum_{i=1}^{n} \alpha_{i} z_{i}<A \bar{u}, e_{1}>+\sum_{i=1}^{n} \beta_{i} z_{i}<A \bar{u}, e_{n}>=A \sum_{i=1}^{n} \alpha_{i} z_{i}=\sum_{i=1}^{n} \alpha_{i} \lambda_{i} z_{i} \\
\left.\sum_{i=1}^{n} \alpha_{i} z_{i}<A \bar{v}, e_{1}>\right)+\sum_{i=1}^{n} \beta_{i} z_{i}<A \bar{v}, e_{n}>=A \sum_{i=1}^{n} \beta_{i} z_{i}=\sum_{i=1}^{n} \beta_{i} \lambda_{i} z_{i}
\end{array}\right. \\
(E)\left\{\begin{array}{l}
\alpha_{i}<A \bar{u}, e_{1}>+\beta_{i}<A \bar{u}, e_{n}>=\alpha_{i} \lambda_{i} \\
\left.\alpha_{i}<A \bar{v}, e_{1}>\right)+\beta_{i}<A \bar{v}, e_{n}>=\beta_{i} \lambda_{i}
\end{array}\right.
\end{gathered}
$$

Under suitable conditions on $A$, the solution of this system can be obtained using iterative method like Jacobi or Gauss-Seidel method as follow:

\section{Algorithm for solving the system:}

For $p=0,1, \ldots$ until convergence solve the following $i$ set of $2 \times 2$ linear systems

$$
\left(E_{p}\right)\left\{\begin{array}{l}
\alpha_{i}^{p+1}<A \bar{u}^{p}, e_{1}>+\beta_{i}^{p+1}<A \bar{u}^{p}, e_{n}>=\alpha_{i}^{p} \lambda_{i} \\
\alpha_{i}^{p+1}<A \bar{v}^{p}, e_{1}>+\beta_{i}^{p+1}<A \bar{v}^{p}, e_{n}>=\beta_{i}^{p} \lambda_{i}
\end{array}\right.
$$




\section{Convergence analysis:}

$\left(E_{p}\right)$ is a $2 x 2$ system equation who have a simple expression as follow:

$$
\Delta_{p}=<A \bar{u}^{p}, e_{1}><A \bar{v}^{p}, e_{n}>-<A \bar{u}^{p}, e_{n}><A \bar{v}^{p}, e_{1}>
$$

is independent of the index $i$ and can be computed only once. Then

$$
\left\{\begin{array}{c}
\alpha_{i}^{p+1}=\frac{1}{\Delta_{p}}\left|\begin{array}{cc}
\alpha_{i}^{p} \lambda_{i} & <A \bar{u}^{p}, e_{n}> \\
\beta_{i}^{p} \lambda_{i} & <A \bar{v}^{p}, e_{n}>
\end{array}\right|=\frac{\lambda_{i}}{\Delta_{p}}\left[\alpha_{i}^{p}<A \bar{v}^{p}, e_{n}>-\beta_{i}^{p}<A \bar{u}^{p}, e_{n}>\right] \\
\beta_{i}^{p+1}=\frac{1}{\Delta_{p}}\left|\begin{array}{cc}
<A \bar{u}^{p}, e_{1}> & \alpha_{i}^{P} \lambda_{i} \\
<A \bar{v}^{p}, e_{1}> & \beta_{i}^{P} \lambda_{i}
\end{array}\right|=\frac{\lambda_{i}}{\Delta_{p}}\left[\beta_{i}^{p}<A \bar{u}^{p}, e_{1}>-\alpha_{i}^{p}<A \bar{v}^{p}, e_{1}>\right]
\end{array}\right.
$$

Let

$$
\begin{gathered}
\gamma_{i, j}^{p+1}=\alpha_{i}^{p+1} \beta_{j}^{p+1}-\alpha_{j}^{p+1} \beta_{i}^{p+1} \\
=\frac{\lambda_{i} \lambda_{j}}{\Delta_{p}^{2}}\left(\alpha_{i}^{p}<A \bar{v}^{p}, e_{n}>-\beta_{i}^{p}<A \bar{u}^{p}, e_{n}>\right)\left(\beta_{j}^{p}<A \bar{u}^{p}, e_{1}>-\alpha_{j}^{p}<A \bar{v}^{p}, e_{1}>\right) \\
-\frac{\lambda_{i} \lambda_{j}}{\Delta_{p}^{2}}\left(\alpha_{j}^{p}<A \bar{v}^{p}, e_{n}>-\beta_{j}^{p}<A \bar{u}^{p}, e_{n}>\right)\left(\beta_{i}^{p}<A \bar{u}^{p}, e_{1}>-\alpha_{i}^{p}<A \bar{v}^{p}, e_{1}>\right)
\end{gathered}
$$

so

$$
\begin{aligned}
& \gamma_{i, j}^{p+1}= \\
& \begin{aligned}
\frac{\lambda_{i} \lambda_{j}}{\Delta_{p}^{2}}\left[( \alpha _ { i } ^ { p } \beta _ { j } ^ { p } - \alpha _ { j } ^ { p } \beta _ { i } ^ { p } ) \left(<A \bar{u}^{p}, e_{1}><A \bar{v}^{p}, e_{n}>-\right.\right. & \left.\left.<A \bar{u}^{p}, e_{n}><A \bar{v}^{p}, e_{1}>\right)\right] \\
& =\frac{\lambda_{i} \lambda_{j}}{\Delta_{p}^{2}} \gamma_{i, j}^{p} \Delta_{p}=\frac{\lambda_{i} \lambda_{j}}{\Delta_{p}} \gamma_{i, j}^{p} .
\end{aligned}
\end{aligned}
$$

Lemma 1. a.

$$
\Delta_{p}=\sum_{l<k} \lambda_{i} \lambda_{i} \gamma_{i, j}^{p+1}\left(<z_{l}, e_{1}><z_{k}, e_{n}>-<z_{k}, e_{1}><z_{l}, e_{n}>\right) .
$$

b.

$$
\gamma_{i, j}^{p+1}=\frac{\left(\lambda_{i} \lambda_{j}\right)^{p+1} \gamma_{i, j}^{0}}{\sum_{l<k}\left(\lambda_{l} \lambda_{k}\right)^{p+1} \gamma_{l, k}^{0} \sigma_{l, k}},
$$

where $\sigma_{l, k}=<z_{l}, e_{1}><z_{k}, e_{n}>-<z_{k}, e_{1}><z_{l}, e_{n}>$ 
Proof. Easy compute gives the results.

Lemma 2. $\forall i, j>2, \lim _{p \rightarrow \infty} \gamma_{i, j}^{p}=0$

Proof. With Lemma1-b, we have

$$
\gamma_{i, j}^{p+1}=\frac{\left(\lambda_{i} \lambda_{j}\right)^{p+1} \gamma_{i, j}^{0}}{\left(\lambda_{1} \lambda_{2}\right)^{p+1} \gamma_{1,2}^{0} \sigma_{1,2}+\sum_{1<l<k}\left(\lambda_{l} \lambda_{k}\right)^{p+1} \gamma_{l, k}^{0} \sigma_{l, k}}
$$

then

$$
\gamma_{i, j}^{p+1}=\frac{\left(\lambda_{i} \lambda_{j}\right)^{p+1} \gamma_{i, j}^{0}}{\left(\lambda_{1} \lambda_{2}\right)^{p+1}\left(\gamma_{1,2}^{0} \sigma_{1,2}+\sum_{1<l<k}\left(\frac{\lambda_{l} \lambda_{k}}{\lambda_{1} \lambda_{2}}\right)^{p+1} \gamma_{l, k}^{0} \sigma_{l, k}\right)}
$$

Suppose that $\gamma_{1,2}^{0}$ and $\sigma_{1,2}$ are both not null, then

$\lim _{p \rightarrow \infty} \gamma_{i, j}^{p}=0$

Lemma 3. For $(i, j)=(1,2)$, we have $\lim _{p \rightarrow \infty} \gamma_{1,2}^{p}=\frac{1}{\sigma_{1,2}}$

Proof. From Lemma 1,

$$
\begin{gathered}
\gamma_{1,2}^{p+1}=\frac{\left(\lambda_{1} \lambda_{2}\right)^{p+1} \gamma_{1,2}^{0}}{\left(\lambda_{1} \lambda_{2}\right)^{p+1}\left(\gamma_{1,2}^{0} \sigma_{1,2}+\sum_{1<l<k}\left(\frac{\lambda_{l} \lambda_{k}}{\lambda_{1} \lambda_{2}}\right)^{p+1} \gamma_{l, k}^{0} \sigma_{l, k}\right)} \\
=\frac{\gamma_{1,2}^{0}}{\gamma_{1,2}^{0} \sigma_{1,2}+\sum_{1<l<k}\left(\frac{\lambda_{l} \lambda_{k}}{\lambda_{1} \lambda_{2}}\right)^{p+1} \gamma_{l, k}^{0} \sigma_{l, k}}
\end{gathered}
$$

with the same hypothesis that $\gamma_{1,2}^{0}$ and $\sigma_{1,2}$ are both not null, then

$$
\lim _{p \rightarrow \infty} \gamma_{1,2}^{p}=\frac{\gamma_{1,2}^{0}}{\gamma_{1,2}^{0} \sigma_{1,2}}=\frac{1}{\sigma_{1,2}}
$$

\section{Error Criteria}

We denote by $\varepsilon_{p}^{1}$ and $\varepsilon_{p}^{2}$ the error calculation defined by

$$
\left\{\begin{array}{l}
\varepsilon_{p}^{1}=\bar{u}^{p+1}-\bar{u}^{p}=\sum_{i=1}^{n} \alpha_{i}^{p+1} z_{i}-\sum_{i=1}^{n} \alpha_{i}^{p} z_{i}=\sum_{i=1}^{n} A_{i} z_{i} \\
\varepsilon_{p}^{2}=\bar{v}^{p+1}-\bar{v}^{p}=\sum_{i=1}^{n} \beta_{i}^{p+1} z_{i}-\sum_{i=1}^{n} \beta_{i}^{p} z_{i}=\sum_{i=1}^{n} B_{i} z_{i}
\end{array}\right.
$$


Let us give the expression of $A_{i}=\alpha_{i}^{p+1}-\alpha_{i}^{p}$ and $B_{i}=\beta_{i}^{p+1}-\beta_{i}^{p}$,

$$
\begin{gathered}
A_{i}=\frac{\lambda_{i}}{\Delta_{p}}\left[\alpha_{i}^{p}<A \bar{v}^{p}, e_{n}>-\beta_{i}^{p}<A \bar{u}^{p}, e_{n}>\right] \\
-\frac{\lambda_{i}}{\Delta_{p-1}}\left[\alpha_{i}^{p-1}<A \bar{v}^{p-1}, e_{n}>-\beta_{i}^{p-1}<A \bar{u}^{p-1}, e_{n}>\right] \\
=\frac{\lambda_{i}}{\Delta_{p}}\left[\sum_{j=1}^{n} \alpha_{i}^{p} \lambda_{j} \beta_{j}^{p}<z_{j}, e_{n}>-\sum_{j=1}^{n} \alpha_{j}^{p} \lambda_{j} \beta_{i}^{p}<z_{j}, e_{n}>\mid-\right. \\
\frac{\lambda_{i}}{\Delta_{p-1}}\left[\sum_{j=1}^{n} \alpha_{i}^{p-1} \lambda_{j} \beta_{j}^{p-1}<z_{j}, e_{n}>-\sum_{j=1}^{n} \alpha_{j}^{p-1} \lambda_{j} \beta_{i}^{p-1}<z_{j}, e_{n}>\right. \\
=\frac{\lambda_{i}}{\Delta_{p}}\left[\sum_{j=1}^{n} \lambda_{j}<z_{j}, e_{n}>\left(\alpha_{i}^{p} \beta_{j}^{p}-\alpha_{j}^{p} \beta_{i}^{p}\right)\right] \\
-\frac{\lambda_{i}}{\Delta_{p-1}}\left[\sum_{j=1}^{n} \lambda_{j}<z_{j}, e_{n}>\left(\alpha_{i}^{p-1} \beta_{j}^{p-1}-\alpha_{j}^{p-1} \beta_{i}^{p-1}\right) \mid\right.
\end{gathered}
$$

then

$$
A_{i}=\sum_{j=1}^{n}<z_{j}, e_{n}>\left(\gamma_{i, j}^{p+1}-\gamma_{i, j}^{p}\right)
$$

and by the Lemma 2, we get

$$
\forall i, j>2 A_{i} \rightarrow 0 \text { where } p \rightarrow \infty
$$

then for the large $p$, we get for $i<j \leq 2$ :

$$
\varepsilon_{p}^{1}=\left(\frac{\lambda_{1}^{2}}{\Delta_{p}}-1\right) \gamma_{1,1}^{p}<z_{1}, e_{n}>+\left(\frac{\lambda_{1} \lambda_{2}}{\Delta_{p}}-1\right) \gamma_{1,2}^{p}<z_{2}, e_{n}>
$$

so

$$
\gamma_{1,1}^{P}=\alpha_{1}^{p} \beta_{1}^{p}-\alpha_{1}^{p} \beta_{1}^{p}=0
$$

we write

$$
\varepsilon_{p}^{1}=\left(\frac{\lambda_{1} \lambda_{2}}{\Delta_{p}}-1\right) \gamma_{1,2}^{p}<z_{2}, e_{n}>=\left(\gamma_{1,2}^{p+1}-\gamma_{1,2}^{p}\right)<z_{2}, e_{n}>
$$


then

$$
\begin{aligned}
& \varepsilon_{p}^{1} \rightarrow 0 \\
& p \rightarrow \infty
\end{aligned}
$$

with the same reasoning, we get :

$$
\begin{aligned}
& \varepsilon_{p}^{2} \rightarrow 0 \\
& p \rightarrow \infty
\end{aligned}
$$

Then, the sequence $\left\{\bar{u}^{p}\right\}_{p}$ and $\left\{\bar{v}^{p}\right\}_{p}$ converge respectively to $\bar{u}=\alpha_{1} z_{1}+\alpha_{2} z_{2}$ and $\bar{v}=\beta_{1} z_{1}+\beta_{2} z_{2}$.

\section{Matrix Factorization}

In this section, we will expressed the double power iteration method as a Quadrant Interlocking Factorization (QIF) which introduced by [7]. For that, we need to repeat our previous work to the transformed matrix $A^{(1)}$ recursively $\left\lfloor p=\frac{n}{2}\right\rfloor$ time step.

Having computing $\bar{u}$ and $\bar{v}$ previously, we compute the matrices $W$ and $Z$ as follow :

$$
\begin{aligned}
& Z_{1,1}^{(1)}=a_{1,1}+<A u, e_{1}> \\
& Z_{n, 1}^{(1)}=a_{n, 1}+<A u, e_{n}>\quad, \\
& Z_{1, n}^{(1)}=a_{1, n}+<A v, e_{1}> \\
& W_{., 1}^{(1)}=\bar{u} \\
& W_{i, i}^{(1)}=1 \\
& W_{., n}^{(1)}=\bar{v} \\
& \left.Z_{n, n}^{(1)}=a_{n, n}+<A v, e_{n}\right\rangle \quad, \\
& W_{i, j}^{(1)}=0 \\
& 2 \leq i, j \leq n-1 \\
& i \neq j
\end{aligned}
$$

and

$$
Z_{i, j}^{(1)}=a_{i, j}-u_{i} a_{1, j}-v_{i} a_{n, j} \quad, \quad 2 \leq i, j \leq n-1 .
$$

The method is clearly recursive and results after $p$ steps in finals matrices $W$ of the form (1)

$$
W=\left(\begin{array}{ccccc}
1 & 0 & \ldots & 0 & 0 \\
w_{2,1} & \ddots & 0 & . & w_{2, n} \\
\vdots & w_{.,} & 1 & w_{., .} & \vdots \\
w_{n-1,1} & . \cdot & 0 & \ddots & w_{n-1, n} \\
0 & 0 & \ldots & 0 & 1
\end{array}\right)
$$


and $Z$ of the form $(2)$

$$
Z=\left(\begin{array}{ccccc}
z_{1,1} & \cdots & z_{i, 1} & \cdots & z_{1, n} \\
0 & \ddots & z_{., .} & . & 0 \\
\vdots & 0 & z_{i, i} & 0 & \vdots \\
0 & . \cdot & z_{., .} & \ddots & 0 \\
z_{1, n} & \cdots & z_{i, n} & \cdots & z_{n, n}
\end{array}\right)
$$

Then, to solve a linear system $A x=b$, where $A=W Z$, we solve the two systems $Z x=y$ and $W y=b$. In the other hand, by expressing $A$ in the form $A=W Z W^{-1}$ we compute the eigenvalues of $A$ using $p$ step factorization : At step $k, 1 \leq k \leq p$, we compute an elementary matrix $W_{k}$ such that $W_{1} \cdots W_{k}$ and $Z_{k}=W_{k}^{-1} Z_{k-1} W_{k}=\left(W_{k} \cdots W_{1}\right)^{-1} A\left(W_{k} \cdots W_{1}\right)$ have the form (2). Application of $W_{k}$ introduces zeros below and before diagonal of $A$ in columns $k$ and $n-k$.

The $W$ matrix is recovered by formatting the product $W_{p} \cdots W_{1}$ and $Z=Z_{p}$. So, at step 1, we calculate

$$
w_{., 1}^{T}=\left(0, w_{2,1}, \ldots, w_{n-1,1}, 0\right), w_{., n}^{T}=\left(0, w_{2, n}, \ldots, w_{n-1, n}, 0\right)
$$

Then, $Z_{1}=W_{1}^{-1} A W_{1}$ imply that $\forall 2 \leq i \leq n-1$.

$$
\left\{\begin{array}{l}
\left.w_{i, 1}\left(a_{1,1}+<A w_{., 1}, e_{1}>\right)+w_{i, n}\left(a_{n, 1}+<A w_{., 1}, e_{1, n}>\right)=a_{i, 1}+<A w_{., 1}, e_{1}>\right) \\
\left.w_{i, 1}\left(a_{1, n}+<A w_{., n}, e_{1}>\right)+w_{i, n}\left(a_{n, n}+<A w_{., n}, e_{n}>\right)=a_{i, n}+<A w_{., n}, e_{1}>\right)
\end{array}\right.
$$

At step 2, we compute

$$
w_{., 2}^{T}=\left(0,0, w_{3,2}, \ldots, w_{n-2,2}, 0,0\right), w_{., n-1}^{T}=\left(0,0, w_{3, n-1}, \ldots, w_{n-2, n-1}, 0,0\right)
$$

to form the $W_{2}$ matrix. Then we compute $Z_{2}=W_{2}^{-1} Z_{1} W_{2}$.

Note that the $W_{2}$ transformation let the first and last columns and the first and last rows of $Z_{1}$ unchanged i.e

$$
z_{1, i}^{(2)}=z_{1, i}^{(1)} \quad, \quad z_{n, i}^{(2)}=z_{n, i}^{(1)} \quad, \quad z_{i, 1}^{(2)}=z_{i, 1}^{(1)} \quad, \quad z_{i, n}^{(2)}=z_{i, n}^{(1)} \quad, \quad \forall 2 \leq i \leq n-1
$$

Hence, we obtain the same result if we let

$$
\tilde{w}_{., 2}^{T}=\left(0, w_{3,2}, \ldots, w_{n-2,2}, 0\right), \tilde{w}_{., n-1}^{T}=\left(0, w_{3, n-1}, \ldots, w_{n-2, n-1}, 0\right)
$$

and

$$
\tilde{W}_{2}=I+\tilde{w}_{., 2} e_{2}^{T}+\tilde{w}_{., n-1} e_{n-1}^{T}
$$


here $e_{2}, e_{n-1}$ are the first and last column of $I_{n-1}$. Next, we compute the $n-1$ by $n-1$ matrix $\tilde{Z}_{2}=\tilde{W}_{2}^{-1} \tilde{Z}_{1} \tilde{W}_{2}$ where $\tilde{Z}_{1}=\left(z_{i, j}^{(1)}\right)_{2 \leq i, j \leq n-1}$.

$$
\begin{gathered}
z_{l, i}^{(k)}=z_{l, i}^{(k-1)}, z_{n-l+1, i}^{(k)}=z_{n-l+1, i}^{(k-1)}, z_{i, l}^{(k)}=z_{i, l}^{(k-1)}, z_{i, n-l+1}^{(k)}=z_{i, n-l+1}^{(k-1)}, \\
\forall 1 \leq i \leq n, 1 \leq l \leq k-1
\end{gathered}
$$

So, at step $k$, it suffice to compute $(n-2 k+2) x(n-2 k+2)$ block of the matrix $Z$ by taken

$$
\begin{gathered}
\tilde{w}_{., k}^{T}=\left(0, w_{k+1, k}, \ldots, w_{n-k, k}, 0\right) \\
\tilde{w}_{., n-k+1}^{T}=\left(0, w_{k+1, n-k+1}, \ldots, w_{n-k, n-k+1}, 0\right) \text { in } \mathbb{R}^{n-2 k+2}
\end{gathered}
$$

The method can be summarized as follow.

$Z_{0}=A$

For $\mathrm{k}=1$ to $\mathrm{p}$

Solve the following systems for $w_{., k}$ and $w_{., k^{\prime}}$ where $k^{\prime}=n-k+1$ and $z_{i, j}$ are the elements of $Z_{k-1}$

$$
\left\{\begin{array}{l}
k+1 \leq i \leq k^{\prime}-1 \\
w_{i, k}\left(z_{k, k}+<Z_{k-1} w_{., k}, e_{k}>\right)+w_{i, k^{\prime}}\left(z_{k^{\prime}, k}\right. \\
\left.+<Z_{k-1} w_{., k}, e_{k^{\prime}}>\right)=z_{i, k}+<Z_{k-1} w_{., k}, e_{i}> \\
w_{i, k}\left(z_{k, k^{\prime}}+<Z_{k-1} w_{., k^{\prime}}, e_{k}>\right)+w_{i, k^{\prime}}\left(z_{k^{\prime}, k^{\prime}}\right. \\
\left.+<Z_{k-1} w_{., k^{\prime}}, e_{k^{\prime}}>\right)=z_{i, k^{\prime}}+<Z_{k-1} w_{., k^{\prime}}, e_{i}>
\end{array}\right.
$$

$W_{k}=I_{k}+w_{., k} e_{k}^{T}+w_{., k^{\prime}} e_{k^{\prime}}^{T} ; Z_{k}=W_{k}^{-1} Z_{k-1} W_{k}$

EndFor

$W=I_{n}+\sum_{k=1}^{p}\left(w_{., k} e_{k}^{T}+w_{., k^{\prime}} e_{k^{\prime}}^{T}\right) ; Z=Z_{p}$

\section{References}

[1] Ipsen, Ilse, and Rebecca M. Wills, Analysis and Computation of Google's PageRank, 7th IMACS International Symposium on Iterative Methods in Scientific Computing, Fields Institute, Toronto, Canada, 5-8 May (2005).

[2] Pankaj Gupta, Ashish Goel, Jimmy Lin, Aneesh Sharma, Dong Wang, and Reza Bosagh Zadeh, WTF: The who-to-follow system at Twitter, Proceedings of the 22nd international conference on World Wide Web (2013).

[3] F. R. Gantmacher, Theory of Matrices II, Chelsea, New York, (1960). DOI: 10.1137/1.9780898718058.

[4] J.H.Wilkinson, The Algebraic Eigenvalue Problem, Oxford University Press, (1965). DOI:10.1137/1008125. 
[5] H. Rautishauser, Solution of eigenvalue problems with LR transformation, N.B.S. Appl. Math. Ser. 49 (1958), 47-81.

[6] Beata Bylina, Solving linear systems with vectorized WZ factorization, Annales UMCS Informatica AI 1 (2003), 5-13.

[7] D. J. Evans, A parallel linear system solver, Inrern. I. Comp. Math. 7 (1979), 227-238. DOI: $10.1080 / 00207167908803174$.

[8] D. J. Evans and A. Hadjidimos, A modification of the quadrant interlocking factorization in parallel method, Inrern. I. Comp. Morh. 8 (1980), 149-166. DOI: 10.1080/00207168008803201.

[9] D. J. Evans, A. Hadjidimos and D. Noutsos, Parallel solution of linear systems by quadrant interlocking factorizalion methods, Computer Methods in Applied Mechanics and Engineering 29 (1981), 97-10\%. DOI: 10.1016/0045-7825(81)90017-7

[10] D. B. O'Leary, P. Whithman, Parallel QR factorization by Householder and modified Gram-Schmidt algorithm, Parallel Computing 16 (1990), 99-112. DOI: 10.1016/01678191(90)90163-4.

[11] J. J. Modi, Parallel algorithms and matrix computation, Oxford Applied Math. and Comput. Science Series (1984).

[12] H. D. Simon, The Lanczos algorithm with partial reorthogonalization, Math. Comput. 42 (1984), 115-142. DOI: 10.2307/2007563.

[13] G. H. Golub, C. F. Van Loan, Matrix computations, The Johns Hopkins Univ Press, (1983). DOI:10.1137/1028073. 
